University of Louisville

ThinkIR: The University of Louisville's Institutional Repository

College of Arts \& Sciences Senior Honors

Theses

College of Arts \& Sciences

$5-2013$

\title{
Homological algebra : Tor functors, Betti numbers, and free resolutions.
}

Ian Philipp

University of Louisville

Follow this and additional works at: https://ir.library.louisville.edu/honors

Part of the Mathematics Commons

\section{Recommended Citation}

Philipp, lan, "Homological algebra : Tor functors, Betti numbers, and free resolutions." (2013). College of Arts \& Sciences Senior Honors Theses. Paper 20.

http://doi.org/10.18297/honors/20

This Senior Honors Thesis is brought to you for free and open access by the College of Arts \& Sciences at ThinkIR: The University of Louisville's Institutional Repository. It has been accepted for inclusion in College of Arts \& Sciences Senior Honors Theses by an authorized administrator of ThinkIR: The University of Louisville's Institutional Repository. This title appears here courtesy of the author, who has retained all other copyrights. For more information, please contact thinkir@louisville.edu. 


\title{
Homological Algebra: Tor Functors, Betti Numbers, and Free Resolutions
}

\author{
By \\ Ian Philipp
}

\author{
Submitted in partial fulfillment of the requirements \\ for Graduation summa cum laude \\ and \\ for Graduation from the Department of Mathematics \\ University of Louisville
}

March 26, 2013 
Many Thanks to my mentor, Dr. Jinjia Li, without whom this would not have been possible and to Dr. Hamid Kulosman for sparking my interest in commutative algebra. 
Philipp

\section{Introduction}

The topic of study for this project is homological algebra, a branch of mathematics with applications to a plethora of other branches of mathematics as well as sensor networks, signal processing, fluid dynamics, particle physics, etc. The amount of literature written on this topic is vast and there are numerous open problems in homological algebra, but this project has a more modest focus. First, we will explain the fundamental terminology with several examples and later we will proceed to some results concerning certain rings and ideals, and then results about modules generated by zero divisors.

\section{Basics}

A ring, denoted $R$, is a mathematical object that generalizes our algebraic intuition about integers (whole numbers). For example, in the integers we may multiply and add without fear of ever producing a number that is not an integer. To be more specific, these operations, addition and mulitplication, are closed. Among the integers there also exist inverses for addition (the negative whole numbers) and special numbers which serve as the additive and multiplicative identities ( 0 and 1$)$. A ring $R$, is just a set (a collection of objects) with a few of these algebraic properties (only a few because we want to consider structures more general and less well-behaved than the integers). Why should we study such an abstract object? For example, when physicists first started doing calculations with the electromagnetic force, the real numbers satisfied their algebraic needs (because there are only two charges, + and - ). But after the discovery of quarks, numbers proved useless because there are six 'flavors' of quarks. In other words, a new algebraic structure was needed to study the interactions of quarks.

Sometimes we are not only interested in the entire ring $R$, but wish to examine substructures. The most interesting substructures are subrings (selfexplanatory) that absorb multiplication. That is, if we have a subring $I$ and an element $a \in I$ and $r \in R$, then $a r \in I$ ( $\in$ indicates that $a$ is an element of $I$ ). If this is true for every combination of elements $a \in I$ and $r \in R$, then we call such $I$ an ideal of $R$. For example, the even numbers form an ideal of the integers (we will denote the integers as $\mathbb{Z}$ from now on) because if $n$ is an even number and $m$ is any integer, then $n m$ is an even number.

In mathematics, it may be quite hard to gather facts (i.e. prove theorems) about very general objects, such as all rings. This occurs because the class of all rings may contain some very bizarre structures which break any patterns we are trying to establish. Therefore it is customary to restrict our studies to 'special' rings. For this project we wish our rings to have two nice properties; both of which make the ring small in certain senses. We want our rings to be such that in every ideal $I$ of $R$, if $a \in I$ then we are able to write $a$ as a linear combination of a specified set of elements $x_{1}, x_{2}, \ldots, x_{n}$. Such a set of elements is said to generate $I$. Therefore we may say that every ideal $I$ of $R$ is finitely 
generated. If a ring $R$ meets this requirement, it is said to be Noetherian.

The other condition we will impose on our rings is slightly more involved. If the ring $R$ in question has a multiplicative identity (for instance, 1 in the integers) then it can be shown that every ideal $I$ is contained in some maximal ideal $\mathfrak{m}$. A ring may have many (infinitely so!) maximal ideals, but we want to study only those rings that have a unique maximal ideal. If this is the case, then a ring is said to be local.

In algebra, often times a structure 'acts' on another structure. For example, the integers can be multiplied by the rational numbers and the result is a rational number. The multiplication in this case is the 'action'. There are also ways to use this idea of 'action' to model the symmetry of geometric objects but we will not discuss this. The structures being acted on are called representations. In particular, if a ring $R$ acts on another structure $M$ via multiplication, this other structure is called an $R$-module. As we said earlier, $\mathbb{Q}$, the set of rational numbers, is a $\mathbb{Z}$-module. Often times $R$-modules can tell you a great deal about the ring itself. A free module is a representation of a ring that consists entirely of (direct sums of) copies of the ring. This is analgous to how the Euclidean space is equivalent to the cartesian product of $\mathbb{R}$, the set of real numbers, with itself, if we add pointwise multiplication and addition.

But nobody studies rings or any other structures in isolation. Often times we are interested in their relationships to one another (as in all other forms of science). The best way to model a relationship is none other than a function. But we are not interested in just any functions, but rather in functions that preserve algebraic structure. Such functions are called homomorphisms. Homomorphisms preserve whichever algebraic properties we are interested in be it rings, modules or otherwise. If $f: A \rightarrow B$ is a homomorphism from $A$ to $B$ then the set $\{a: a \in A$ such that $f(a)=0\}$ is called the kernel of $f$ (denoted $\operatorname{ker}(f))$ and the set $\{b: b \in B, \exists a \in A$ such that $f(a)=b\}$ is called the image of $f$ (denoted $\operatorname{Im}(f))$.

Suppose now that we have a ring $R$ and let $A, B$ and $C$ be $R$-modules. Consider the following diagram

$$
0 \stackrel{f_{1}}{\longrightarrow} A \stackrel{f_{2}}{\longrightarrow} B \stackrel{f_{3}}{\longrightarrow} C \stackrel{f_{4}}{\longrightarrow} 0
$$

where the arrows represent $R$-module homomorphisms. Such a sequence is called short and if $\operatorname{Im}\left(f_{i}\right)=\operatorname{ker}\left(f_{i+1}\right)$ for all $i$ then it is also called exact. Such sequences give a great deal of information about the modules involved.

Finally we may now discuss the term homological. If a sequence $C$ • of the form

$$
\cdots \stackrel{d_{n-2}}{\longrightarrow} C_{n-1} \stackrel{d_{n-1}}{\longrightarrow} C_{n} \stackrel{d_{n}}{\longrightarrow} C_{n+1} \stackrel{d_{n+1}}{\longrightarrow} \cdots
$$

has the property that $\operatorname{Im}\left(d_{i}\right) \subset \operatorname{ker}\left(d_{i+1}\right)$ for all $i$, the sequence is said to be a chain complex. We call the module

$$
H_{n}\left(C_{\bullet}\right)=\frac{\operatorname{ker}\left(d_{n}\right)}{\operatorname{Im}\left(d_{n-1}\right)}
$$

the $n$th homology module of $C_{\boldsymbol{\bullet}}$. The fraction above is the quotient structure of two algebraic structures. One can (very loosely) think of this division as 
Philipp

reducing all the elements of the denominator to 0 in the module that is in the numerator. The homology modules measure how close $C$ • is to being an exact complex. Thus homological algebra is the study of how close chain complexes are to being exact.

This is a fairly abstract subject matter, and at first is a seemingly useless idea, but remember to keep in mind the list of topics that use homological methods mentioned in the introduction. Also, there were numerous statements about rings that remained unsolved for several decades before the advent of homological methods. It is a powerful system of thought indeed.

\section{Fundamentals of Tor}

In this section we wish to discuss the homological structure Tor so that the reader may appreciate this project more fully.

We almost have all the ideas necessary to discuss Tor except two key components, the tensor product and free resolutions. The tensor product is a fairly difficult concept but one can think of it as a way to create new modules from old modules in such a way that some algebraic properties from the old modules are preserved. For example, let $\mathbb{R}[x]$ be all the polynomials with real coeffecients in one variable $x$ and let $\mathbb{R}[y]$ denote the same structure except with the variable $y$. Then the tensor product of $\mathbb{R}[x]$ and $\mathbb{R}[y]$ denoted $\mathbb{R}[x] \otimes \mathbb{R}[y]$ is actually equal to $\mathbb{R}[x, y]$, all the polynomials in two variables with real coeffecients. This is not true for more general structures but is a good motivation.

The Tor modules measure how far from being exact the tensor product $A \otimes-$ is for some fixed module $A$.

A free resolution of an $R$-module $M$ is an exact chain complex of the form

$$
\cdots \longrightarrow F_{1} \longrightarrow F_{0} \longrightarrow M \longrightarrow 0
$$

where each $F_{i}$ is a free module (A vector space is simply a free module over a field). Free resolutions measure how far $M$ is from being a free module and may be infinite. The minimal free resolution of a module is one in which the rank of each free module in the resolution is minimal. The number of copies (in the direct sum sense) of a free module $F$ is of $R$ is denoted $\operatorname{rank}(F)$. Rank is analgous to the dimension of a vector space. The length of the minimal free resolution for each module $M$ is unique and is called the projective dimension of $M$, denoted $\operatorname{pd}(M)$. In a minimal free resolution the ranks are unique and are denoted $\beta_{n}^{R}(M)$, the Betti numbers of $M$.

Now for concreteness and for clarity, instead of defining Tor abstractly we compute $\operatorname{Tor}_{n}^{\mathbb{Z}}\left(\mathbb{Z}_{2}, \mathbb{Z}_{2}\right)$. That is, we compute all the Tor modules where the ring is $\mathbb{Z}$ and where $\mathbb{Z}_{2}$ is the set of integers modulo 2 . More specifically $\mathbb{Z}_{2}=\{0,1\}$ where multiplication is as usual and we define $1+1=0$.

First we find a free resolution of $\mathbb{Z}_{2}$

$$
\cdots \longrightarrow 0 \longrightarrow \mathbb{Z} \stackrel{\times 2}{\longrightarrow} \mathbb{Z} \stackrel{\pi}{\longrightarrow} \mathbb{Z}_{2} \longrightarrow 0
$$

Next we remove $\mathbb{Z}_{2}$ and apply $-\otimes \mathbb{Z}_{2}$ to the sequence. Thus by the flatness of $\mathbb{Z}_{2}$ over $\mathbb{Z}$ we have 


$$
0 \longrightarrow \mathbb{Z} \otimes \mathbb{Z}_{2} \stackrel{\times 2 \otimes 1_{\mathbb{Z}_{2}}}{\longrightarrow} \mathbb{Z} \otimes \mathbb{Z}_{2} \longrightarrow 0
$$

Now there is an identity that $R \otimes_{R} M=M$. So we have

$$
0 \longrightarrow \mathbb{Z}_{2} \stackrel{f}{\longrightarrow} \mathbb{Z}_{2} \longrightarrow 0
$$

One can deduce that $f$ is essentially multiplying by 2 and therefore maps everything to 0 . Now we take the homology modules which in this case are Tor modules. We have

$$
\begin{gathered}
\operatorname{Tor}_{0}^{\mathbb{Z}}\left(\mathbb{Z}_{2}, \mathbb{Z}_{2}\right)=\mathbb{Z}_{2} \otimes \mathbb{Z}_{2} \\
\operatorname{Tor}_{1}^{\mathbb{Z}}\left(\mathbb{Z}_{2}, \mathbb{Z}_{2}\right)=\frac{\operatorname{ker}(0)}{\operatorname{Im}(0)}=\mathbb{Z}_{2} \\
\operatorname{Tor}_{n}^{\mathbb{Z}}\left(\mathbb{Z}_{2}, \mathbb{Z}_{2}\right)=0 \text { for } n>2
\end{gathered}
$$

\section{Results Related to Tor Functors}

For some context, we mention a theorem of Goto and Suzuki [4] that states if $I$ is a nonprincipal ideal and $\operatorname{Tor}_{1}(I, R / I)$ is a free $R / I$-module then $\operatorname{rank}\left[\operatorname{Tor}_{1}(I, R / I)\right]$ is equal to the second Betti number of $R / I$. A special case of the above result says if $\operatorname{Tor}_{1}(I, R / I)=0$ then the second Betti number $\beta_{2}$ of $R / I$ is 0 . In other words, the vanishing of $\operatorname{Tor}_{1}(I, R / I)$ implies $\operatorname{pd}(R / I) \leq 1$ for a non principal ideal. We wondered if it is possible that $\operatorname{Tor}_{1}(I, R / I)$ vanishes and $\operatorname{pd}(R / I)>1$ for some principal ideal $I$. This was proved in the affirmitive.

Let $R=k[x, y]_{(x, y)} /\left(y^{2}, x y\right)$ and $I=\left(x+\left(y^{2}, x y\right)\right)$ where $k$ is a field. Then $\mathrm{R}$ is a Noetherian local ring and $\mathrm{I}$ is a principal ideal generated by a zero divisor. To calculate $\operatorname{Tor}_{1}(I, R / I)$ we construct a minimal free resolution as follows. Map $R$ onto $I$ where $R$ is the free module of rank 1 such that $1 \mapsto x+\left(y^{2}, x y\right)$. The kernel is $Z_{0}=\left(y+\left(y^{2}, x y\right)\right)$. Thus we map another free module of rank one onto $Z_{0}$ by $1 \mapsto y+\left(y^{2}, x y\right)$. The kernel of this map is $Z_{1}=\left(x+\left(y^{2}, x y\right), y+\left(y^{2}, x y\right)\right)$. Since this ideal is generated by two elements we create a map $R^{2} \rightarrow Z_{1}$ defined by $(1,0) \mapsto x$ and $(0,1) \mapsto y$ (from now on we shall abbreviate $x+\left(y^{2}, x y\right)$ to $x$ ). We now inductively build the rest of the resolution as follows:

Definition 1. The Fibonacci numbers are the unique sequence of integers such that $a_{n}=a_{n-1}+a_{n-2}$ with $a_{0}=1$ and $a_{1}=1$.

Proposition 1. Let $F_{\bullet}$ be the minimal free resolution of $I$ and $a_{n}$ be the nth Fibonacci number, then for $n \geq 0, F_{n}=R^{a_{n}}$. The differentials $d_{n}$ of the complex are matrices where each row is a generator of $Z_{n}$ (the $n$th kernel).

Remark. The differential is obvious because this construction is a minimal free resolution.

Proof. We proceed by induction on $n$. The base case was covered in the preceding paragraph, so now we suppose that the rank of $F_{n}$ is equal to $a_{n}$ and wish to show that the rank of $F_{n+1}$ is $a_{n+1}$. Note that the rank of $F_{k}$ is equal to the number of generators of $Z_{k}$ for all $k$ by construction. Therefore, equivalently, 
we shall show that the number of generators of $Z_{n+1}$ is $a_{n+1}$. Let $b_{n+1}$ be the number of generators of $Z_{n+1}$.

The paragraph before proposition 1 shows that the generators of the first few kernels in the resolution are

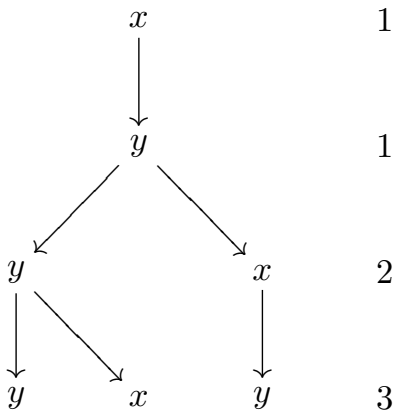

where each row represents the generators of the $n$th kernel and the numbers on the right emphasize that this is indeed the beginning of the Fibonacci sequence. Note that in the diagram when two $y$ 's appear in the generating set of $Z_{3}$, these elements actually represent $(y, 0)$ and $(0, y)$ in the actual free module.

To prove the original assertion we will show that the number of $y$ 's in the generating set of $Z_{n+1}$ is equal to $b_{n}$ and that the number of $x$ 's in the generating set of $Z_{n+1}$ is equal to $b_{n-1}$. We will use induction for this as well.

Let $\alpha_{n}, \omega_{n}$ be the number of $y$ 's and $x$ 's in the generating set of $Z_{n}$ respectively, and assume $\alpha_{n}=b_{n-1}$ and $\omega_{n}=b_{n-2}$ for the inductive hypothesis (the diagram shows the base case). Since each $y$ is annihilated by $x$ or $y$ (because of the specified ring) we have that each $y$ in the generating set of $Z_{n}$ will produce a row containing just an $x$ or $y$ and the remaining entries 0 in the differential $d_{n+1}$. Therefore we may think of $y$ in the generating set of $Z_{n}$ as contributing an $x$ and a $y$ to the generating set of $Z_{n+1}$. Similarly, $x$ will contribute a $y$ to the generating set of $Z_{n+1}$. Therefore every element of the generating set of $Z_{n}$ contributes a $y$ to the generating set of $Z_{n+1}$. Hence we have that $\alpha_{n+1}=b_{n}$ and similarly $\omega_{n+1}=b_{n-1}$. Since this accounts for all the elements in the generating set of $Z_{n+1}$ because it is a kernel we have that $b_{n+1}=\alpha_{n+1}+\omega_{n+1}=b_{n}+b_{n-1}$. This is the Fibonacci recurrence relation.

From the original induction hypothesis we have that, the initial conditions of the recurrence match the Fibonacci sequence, we have that indeed $b_{n}=a_{n}$.

$F_{\bullet}:$

By the last proposition we have that

$$
\cdots \stackrel{d_{4}}{\longrightarrow} R^{3} \stackrel{d_{3}}{\longrightarrow} R^{2} \stackrel{d_{2}}{\longrightarrow} R \stackrel{d_{1}}{\longrightarrow} R \stackrel{d_{0}}{\longrightarrow} I \longrightarrow 0
$$

Now we take the truncated resolution of the complex and apply $-\otimes_{R} \frac{R}{I}$ :

$$
\ldots \stackrel{d_{4} \otimes 1}{\longrightarrow}\left(\frac{R}{I}\right)^{3} \stackrel{d_{3} \otimes 1}{\longrightarrow}\left(\frac{R}{I}\right)^{2} \stackrel{d_{2} \otimes 1}{\longrightarrow}\left(\frac{R}{I}\right) \stackrel{d_{1} \otimes 1}{\longrightarrow}\left(\frac{R}{I}\right) \longrightarrow 0
$$

Let $d_{n}^{*}=d_{n} \otimes_{R} 1$. Then we compute $\operatorname{ker}\left(d_{1}^{*}\right)$ and $\operatorname{Im}\left(d_{2}^{*}\right)$. Since $\frac{R}{I}=$ $k[x, y]_{(x, y)} /\left(y^{2}, x\right)$ we have that the kernel of $d_{1}^{*}$ is just the ideal generated by $y$. For $\operatorname{Im}\left(d_{2}^{*}\right)$, we compute the following. Let $a_{1}, a_{2}, b_{1}, b_{2} \in k$ 


$$
\left(a_{1}+a_{2} y, b_{1}+b_{2} y\right)\left(\begin{array}{l}
x \\
y
\end{array}\right)=x\left(a_{1}+a_{2} y\right)+y\left(b_{1}+b_{2} y\right)=b_{1} y
$$

Hence we have that $\operatorname{Im}\left(d_{2}^{*}\right)$ is also the ideal generated by y. (Note: in the computation there should be fractions since it is a localized ring and cosets since it is also a quotient ring but those have been left out for simplicity.) Since these two sets are the same, $\operatorname{Tor}_{1}(I, R / I)=0$. Also, because the Betti numbers of $R / I$ are the Fibonacci sequence and the resolution is minimal, we have that $\operatorname{pd}(R / I)=\infty$. Hence we have proved

Proposition 2. There exists a ring $R$ and an ideal $I$ such that $\operatorname{Tor}_{1}(I, R / I)=0$ vanishes but $p d(R / I)>1$.

\section{Free Resolutions of Ideals Generated by Zero Divisors}

In the last section, the ideal $I=\left(x+\left(y^{2}, x y\right)\right)$ had the following two properties.

1. $I$ is generated by a zerodivisor

2. $\operatorname{pd}(I)=\infty$

The main question of this paper concerns the following connection between these two properites, namely, does the first always imply the second? We first shall develop the properties of prime ideals necessary for our investigation then shall work toward an answer to this question. A prime ideal is an ideal $\mathfrak{p}$ such that if $r s \in \mathfrak{p}$ where $r, s \in R$, then either $r \in \mathfrak{p}$ or $s \in \mathfrak{p}$. It was a large step in the development of algebra when instead of considering prime elements of a ring, mathematicians began to consider the set of their prime ideals.

To begin, we introduce the idea of localization. We shall use the definition from [3] which we repeat here for reference:

Definition 2. A set $S \subset R$ is multiplicatively closed if for all $x, y \in S$ we have that $x y \in S$. Also $1 \in S$.

Definition 3. Suppose that $S$ is a multiplicateively closed set in $A$ and that $f: A \rightarrow B$ is a ring homomorphism satisfying the two conditions

1. $f(x)$ is a unit of $B$ for all $x \in S$

2. if $g: A \rightarrow C$ is a homomorphism of rings taking every element of $S$ to a unit of $C$ then there exists a unique homomorphism

$$
h: B \rightarrow C \text { such that } g=h f ;
$$

then $B$ is uniquely determined up to isomorphism, and is called the localization or the ring of fractions of $A$ with respect to $S$. We write $B=S^{-1} A$ or $A_{S}$, and call $f: A \rightarrow A_{S}$ the canonical map. 
In [3] it is also shown that the localization can be constructed by defining an equivalence relation on $R \times S$. Let $(a, s) \sim(b, u)$ if there exists $t \in S$ such that $t(a u-b s)=0$. As a notation we let $a / s:=(a, s)$ and define addition and multiplication for fractions as usual. This construction is shown to have the properties in Definition 1. We first show that localization is exact.

Lemma 5.1. Let $R$ be a commutative ring with multiplicative identity and

$$
0 \longrightarrow A \longrightarrow B \longrightarrow C \longrightarrow 0
$$

be a short exact sequence of $R$-modules. If $S$ is a multiplicatively closed set in $R$ then localization with respect to $S$ is an exact functor.

Proof. From Theorem 4.4 of [3] we have that the localization functor is naturally isomorphic to $-\otimes_{R} R_{S}$. It is well known that the tensor product is right exact so we only need to show that localization preserves injections. In other words we need to show that

$$
0 \longrightarrow A \otimes_{R} R_{S} \longrightarrow B \otimes_{R} R_{S}
$$

is exact. First, note that the following diagram commutes:

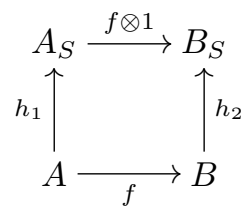

where $f$ is the injection from the exact sequence in the proposition and $h_{1}$ and $h_{2}$ are the corresponding canonical maps. Now suppose that $\frac{x}{s} \in A_{S}$ and $(f \otimes 1)\left(\frac{x}{s}\right)=0$. We claim that $f(x) \in \operatorname{ker}\left(h_{2}\right)$. Observe

$$
h_{2}(f(x))=\frac{f(x)}{1}=f(x) \otimes 1=f(x) \otimes \frac{s}{s}=s\left(f(x) \otimes \frac{1}{s}\right)=0 .
$$

where the second equal sign is identifying the localization with the tensor product. It is standard from the construction of localization described in [3], that if $g$ is a canonical localization map then

$$
\operatorname{ker} g=\{a \in R: \text { there exists } s \in S \text { with } s a=0\} .
$$

Therefore there exists $t \in S$ such that $t f(x)=0$. It is easy to see that, $t$ annihilates $x$ since $f$ is a homomorphism. To be explicit

$$
t f(x)=f(t x)=0
$$

and since $f$ is injective we have that $t x=0$. Thus we have that $x \in \operatorname{ker}\left(h_{1}\right)$ so that $\frac{x}{s}=h_{1}(x)\left(\frac{1}{s}\right)=0$. Hence we have shown that localization is exact.

Remark. Note that we have also shown that $R_{S}$ is a flat module

Suppose $M$ is an $R$-module and that $x \in M$. We call the set

$$
\operatorname{ann}(x)=\{y \in R: y x=0\} .
$$


the annihilator of $x$. Let $M$ be a an $R$-module. A prime ideal $\mathfrak{p}$ is an associated prime ideal of $M$ if $\mathfrak{p}$ is the annihilator $\operatorname{ann}(x)$ of some $x \in M$. The set of all associated prime ideals of a module $M$ is denoted $\operatorname{Ass}(M)$. We need this for the following

Lemma 5.2. If $\mathfrak{p} \in A s s(R)$ then there exists an injective homomorphism $f$ : $\frac{R}{\mathfrak{p}} \rightarrow R_{\mathfrak{p}}$ where $R_{\mathfrak{p}}$ is the localization of $R$ with respect to $S=R-\mathfrak{p}$.

Proof. Note: One can think of $R_{\mathfrak{p}}$ as the set of fractions $\left\{\frac{r}{s}: r \in R, s \notin \mathfrak{p}\right\}$ with the same operations as $\mathbb{Q}$. Define $f: \frac{R}{\mathfrak{p}} \rightarrow R_{\mathfrak{p}}$ by

$$
r+\mathfrak{p} \mapsto \frac{x r}{1}
$$

where $x$ is chosen such that $\mathfrak{p}=\operatorname{ann}(x)$. First we check that $f$ is well-defined. Suppose we have that $r+\mathfrak{p}=r^{\prime}+\mathfrak{p}$. Then there exists $p \in \mathfrak{p}$ such that $r-r^{\prime}=p$. Hence we have that $r=r^{\prime}+p$. Therefore

$$
f(r+\mathfrak{p})=f\left(r^{\prime}+p+\mathfrak{p}\right)=\frac{x\left(r^{\prime}+p\right)}{1}=\frac{x r^{\prime}+x p}{1}=\frac{x r^{\prime}}{1}=f\left(r^{\prime}+\mathfrak{p}\right)
$$

Thus $f$ is a well-defined function. Now we check that $f$ is an $R$-module homomorphism. If $r, s \in R$ then

$$
\begin{gathered}
f[(r+\mathfrak{p})+(s+\mathfrak{p})]=f(r+s+\mathfrak{p})=\frac{x(r+s)}{1}=\frac{x r+x s}{1}=f(r+\mathfrak{p})+f(s+\mathfrak{p}) \\
f(r(s+\mathfrak{p}))=f(r s+\mathfrak{p})=\frac{x r s}{1}=\frac{r(x s)}{1}=r f(s+\mathfrak{p})
\end{gathered}
$$

Lastly we check that $f$ is injective. Suppose that $f(y+\mathfrak{p})=0$. This implies that $\frac{x y}{1}=0$. This is only true if there exists $t \notin \mathfrak{p}$ (by the definition of localization) such that $t x y=0$ or $x(y t)=0$. Since $\mathfrak{p}=\operatorname{ann}(x)$ we have that $y t \in \mathfrak{p}$ and since $\mathfrak{p}$ is a prime ideal then $y \in \mathfrak{p}$. Hence we have that $y+\mathfrak{p}=\mathfrak{p}=0_{\frac{R}{\mathfrak{p}}}$.

Now we will briefly describe the construction of an object similar to Tor. Let $A, B$ be $R$-modules and define $\operatorname{Hom}_{R}(A, B)$ to be the set of all $R$-module homomorphisms from $A$ to $B$. Similarly to how we defined Tor (take a free resolution, apply $-\otimes B$, then take cohomology) we define $\operatorname{Ext}_{R}^{i}(A, B)$ (take a projective resolution of $A$, apply $\operatorname{Hom}(-, B)$, then take homology).

Remark. If one is knowledgable of injective modules one can also define $\operatorname{Ext}_{R}^{i}(A, B)$ by taking an injective resolution of $B$, applying $\operatorname{Hom}(A,-)$ and then taking cohomology on this sequence.

The following definition is used frequently in commutative algebra. The $I$-depth of a ring $R$ is defined as

$$
\operatorname{depth}(I, R)=\min \left\{i: \operatorname{Ext}_{R}^{i}\left(\frac{R}{I}, R\right) \neq 0\right\}
$$


If $I=\mathfrak{m}$, the maximal ideal of a local $\operatorname{ring} R$, then we write $\operatorname{depth}(\mathfrak{m}, R)=$ $\operatorname{depth}(R)$. A regular sequence is a sequence $x_{1}, x_{2}, \ldots, x_{n}$ of elements of $R$ in which $\left(x_{1}, x_{2}, \ldots, x_{n}\right) \neq R$ and $x_{i}$ is a nonzerodivisor of

$$
\frac{R}{\left(x_{1}, x_{2}, \ldots, x_{i-1}\right) R}, \text { for } i=1,2, \ldots, n \text {. }
$$

It can be shown that the length of all maximal regular sequences are equal and that the length of a maximal sequence is equal to $\operatorname{depth}(R)$ [1]. All this is necessary for the following lemma

Lemma 5.3. If $\mathfrak{p} \in A \operatorname{ss}(R)$ then $\operatorname{depth}\left(R_{\mathfrak{p}}\right)=0$.

Proof. We have that the $\operatorname{depth}(\mathfrak{p}, R)=0$ since $\mathfrak{p} \in \operatorname{Ass}(R)$ (hence $\mathfrak{p}$ contains only zerodivisors). The $\operatorname{depth}\left(R_{\mathfrak{p}}\right)$ is equal to the length of the longest regular sequence in $\mathfrak{p} R_{\mathfrak{p}}$, the maximal ideal of $R_{\mathfrak{p}}$. But every element of $\mathfrak{p} R_{\mathfrak{p}}$ annihilates $\frac{x}{1}$ where $\mathfrak{p}=\operatorname{ann}(x)$. Hence $\operatorname{depth}(\mathfrak{p}, R)=\operatorname{depth}\left(R_{\mathfrak{p}}\right)=0$.

Remark. An alternate proof of lemma 5.3 is possible since $\operatorname{Ext}^{0}(A, B)=\operatorname{Hom}(A, B)$ [5] and we know $\operatorname{Hom}_{R}\left(\frac{R}{\mathfrak{p}}, R\right) \neq 0$ by slightly adjusting the proof of lemma 5.2. Therefore we know that $\operatorname{depth}(P, R)=0$ and can show that $\operatorname{Hom}_{R}\left(\frac{R}{\mathfrak{p}}, R\right)_{\mathfrak{p}} \neq 0$. This is not hard as lemma 5.2 induces a homomorphism in localization and that localization commutes with quotients.

We prove yet another lemma necessary for the theorem.

Lemma 5.4. Let

$$
0 \longrightarrow F_{n} \longrightarrow F_{n-1} \longrightarrow \cdots \longrightarrow F_{2} \longrightarrow F_{1} \longrightarrow 0
$$

be an exact sequence of free modules and $n \geq 2$. Then

$$
\sum_{i=1}^{n}(-1)^{i} \operatorname{rank}\left(F_{i}\right)=0
$$

Proof. We proceed by induction on $n$. When $n=2$ we have

$$
0 \longrightarrow F_{2} \longrightarrow F_{1} \longrightarrow 0
$$
have

which implies that $F_{2} \cong F_{1}$ and $\operatorname{so} \operatorname{rank}\left(F_{2}\right)=\operatorname{rank}\left(F_{1}\right)$. When $n=3$ we

$$
0 \longrightarrow F_{3} \longrightarrow F_{2} \longrightarrow F_{1} \longrightarrow 0
$$

so the sequence splits since $F_{1}$ is free [5] and $\operatorname{rank}\left(F_{2}\right)=\operatorname{rank}\left(F_{3}\right)+\operatorname{rank}\left(F_{1}\right)$.

Now suppose that the statement is true for $n-1$ and let

$$
0 \longrightarrow F_{n} \longrightarrow F_{n-1} \longrightarrow \cdots \longrightarrow F_{2} \longrightarrow F_{1} \longrightarrow 0
$$

be an exact sequence of free modules. Let $K$ be the kernel of the homomorphism $F_{2} \rightarrow F_{1}$ in the exact sequence. We then have the commutative diagram: 
The sequence

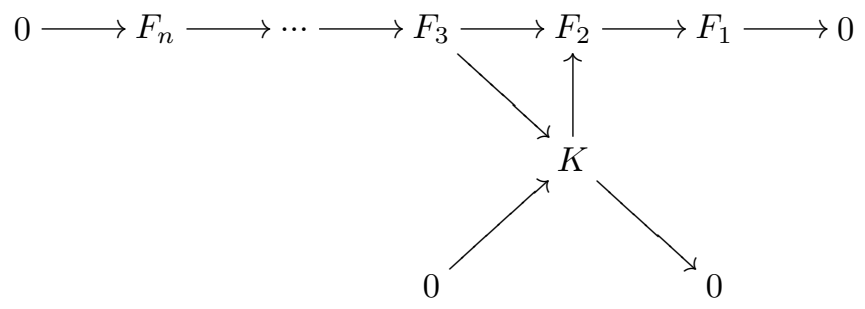

$$
0 \longrightarrow \mathrm{K} \longrightarrow \mathrm{F}_{2} \longrightarrow \mathrm{F}_{1} \longrightarrow 0
$$

splits, again because $F_{1}$ is free. Hence $K$ is a free module with $\operatorname{rank}(K)=$ $\operatorname{rank}\left(F_{2}\right)-\operatorname{rank}\left(F_{1}\right)$. Also

$$
0 \longrightarrow F_{n} \longrightarrow \cdots \longrightarrow F_{3} \longrightarrow K
$$

is an exact sequence of $n-1$ free modules, so by the induction hypothesis

$$
\sum_{i=3}^{n}(-1)^{i} \operatorname{rank}\left(F_{i}\right)+\operatorname{rank}(K)=0
$$

which implies that

$$
\sum_{i=3}^{n}(-1)^{i} \operatorname{rank}\left(F_{i}\right)+\operatorname{rank}\left(F_{2}\right)-\operatorname{rank}\left(F_{1}\right)=\sum_{i=1}^{n}(-1)^{i} \operatorname{rank}\left(F_{i}\right)=0
$$

as desired.

We need a lemma, known as prime avoidance, in order to prove the final lemma for our theorem.

Lemma 5.5. Let $I \subset R$ be an ideal and $\mathfrak{p}_{1}, \mathfrak{p}_{2}, \ldots, \mathfrak{p}_{n}$ be prime ideals. If $I \subset$ $\cup_{i=1}^{n} \mathfrak{p}_{i}$, then there exists $i$ such that $I \subset \mathfrak{p}_{i}$.

Proof. We proceed by induction on $n$. If $n=1$ the statement is trivial. Suppose $n=2$ and to show a contradiction that $I$ is not a proper subset of either $\mathfrak{p}_{1}$ or $\mathfrak{p}_{2}$. Then there exists $x \in I-\mathfrak{p}_{1}$ and $y \in I-\mathfrak{p}_{2}$ by our assumption that $I$ is not properly contained in either $\mathfrak{p}_{1}$ or $\mathfrak{p}_{2}$. The element $x+y \in I$ because $I$ is an ideal but it is not in either $\mathfrak{p}_{1}$ or $\mathfrak{p}_{2}$, a contradiction.

Therefore now assume that $I \subset \cup_{i=1}^{n} \mathfrak{p}_{i}$. We may assume that $I$ is not properly contained in the union of any $n-1$ element subset of $\left\{\mathfrak{p}_{1}, \mathfrak{p}_{2}, \ldots, \mathfrak{p}_{n}\right\}$ or by the induction hypothesis we are finished. Therefore we have that there exists $x \in I-\cup_{i=1}^{n-1} \mathfrak{p}_{i}$ implying that $x \in \mathfrak{p}_{n}$. Let us also take $x_{i} \in \mathfrak{p}_{i}$ with $x_{i} \notin \mathfrak{p}_{i} \cup \mathfrak{p}_{n}$ (this is possible by the case $\mathrm{n}=2$ ) and with $x_{i} \in I$ for $i<n$ and consider the element $y=x_{1} x_{2} \ldots x_{n-1}+x$. Since $\mathfrak{p}_{n}$ is prime $x_{1} x_{2} \ldots x_{n-1} \notin \mathfrak{p}_{n}$ and so $y \in I$ but $y$ is not contained in any $\mathfrak{p}_{i}$. Contradiction.

and this brings us to the last lemma.

Lemma 5.6. Let $R$ be a Noetherian ring. If $M_{\mathfrak{p}}=0$ for every $\mathfrak{p} \in \operatorname{Ass}(R)$. Then $\operatorname{ann}(M) \not \subset \cup_{\mathfrak{p} \in A s s(R)} \mathfrak{p}$. 
Proof. Assume that $\operatorname{ann}(M) \subset \cup_{\mathfrak{p} \in \operatorname{Ass}(R)^{\mathfrak{p}}}$. Since $R$ is Noetherian, $\operatorname{Ass}(R)$ is finite (for suppose not, to find an ideal that is not finitely generated take $I=\left(x_{1}, x_{2}, \ldots\right)$ where $\left.\mathfrak{p}_{\mathfrak{i}}=\operatorname{ann}\left(x_{i}\right)\right)$. By lemma 5.5 we have that there exists $\mathfrak{q} \in \operatorname{Ass}(R)$ such that $\operatorname{ann}(M) \subset \mathfrak{q}$. If $M_{\mathfrak{q}}=0$, assume $M=\left(u_{1}, \ldots, u_{n}\right)$. Then there exists $r_{i} \in R-\mathfrak{q}$ such that $r_{i} u_{i}=0, i=1,2, \ldots, n$. Then $r=\prod_{i=1}^{n} r_{i} \in$ $\operatorname{ann}(M)$ but not in $\mathfrak{q}$, a contradiction.

Now we can prove the main theorem

Theorem 5.7. Let $R$ be a Noetherian local ring and $M$ be an $R$-module with finite projective dimension. Then $\operatorname{ann}(M)=0$ or ann $(M)$ contains a nonzerodivisor on $R$.

Proof. Let

$$
0 \longrightarrow F_{n} \longrightarrow \cdots \longrightarrow F_{2} \longrightarrow F_{1} \longrightarrow M \longrightarrow 0
$$

be a minimal free resolution of $M$, which exists because $R$ is local. Since $R$ is Noetherian, $\operatorname{Ass}(R) \neq \emptyset[3]$. Therefore by localizing at $\mathfrak{p} \in \operatorname{Ass}(R)$, we obtain

$$
0 \longrightarrow\left(F_{n}\right)_{\mathfrak{p}} \longrightarrow \cdots\left(F_{2}\right)_{\mathfrak{p}} \longrightarrow\left(F_{1}\right)_{\mathfrak{p}} \longrightarrow(M)_{\mathfrak{p}} \longrightarrow 0
$$

by lemma 5.1. Since we chose $\mathfrak{p}$ to be an associated prime of $R$, we have that $\operatorname{depth}\left(R_{\mathfrak{p}}\right)=0$ by lemma 5.3. We now use the famous Auslander-Buchsbaum Formula (which can be found in [5] for example) which states for any Noetherian local ring $R^{\prime}$ and $N$ an $R^{\prime}$-module of finite projective dimension the following:

$$
\operatorname{pd}_{R^{\prime}}(N)+\operatorname{depth}_{R^{\prime}}(N)=\operatorname{depth}\left(R^{\prime}\right) .
$$

For our case this implies,

$$
\operatorname{pd}_{R_{\mathfrak{p}}}\left(M_{\mathfrak{p}}\right)+\operatorname{depth}_{R_{\mathfrak{p}}}\left(M_{\mathfrak{p}}\right)=0 .
$$

Since both quantities on the left hand side are greater than or equal to zero, both must be zero. This implies that $M_{\mathfrak{p}}$ is projective and hence free since $R$ is a local ring (projecitve modules have projective dimension $0[5]$ ). Therefore the sequence

$$
0 \longrightarrow\left(F_{n}\right)_{\mathfrak{p}} \longrightarrow \cdots \longrightarrow\left(F_{2}\right)_{\mathfrak{p}} \longrightarrow\left(F_{1}\right)_{\mathfrak{p}} \longrightarrow M_{\mathfrak{p}} \longrightarrow 0
$$

is an exact sequence of free $R_{\mathfrak{p}}$-modules. Since the localization of free modules are also free over $R_{\mathfrak{p}}$ (this is trivial using the fact that localization is naturally isomorphic to $-\otimes_{R} R_{\mathfrak{p}}$ and that the tensor commutes with direct sums) we have that

$$
\sum_{i=1}^{n}(-1)^{i} \operatorname{rank}\left(F_{i}\right)=\operatorname{rank}\left(M_{\mathfrak{p}}\right)
$$

by lemma 5.4 .

We now have two cases depending upon whether or not $\operatorname{rank}\left(M_{\mathfrak{p}}\right)=0$. Case 1. Suppose that $\operatorname{rank}\left(M_{\mathfrak{p}}\right)=0$ for all $\mathfrak{p} \in \operatorname{Ass} R$. This implies that $M_{\mathfrak{p}}=0$ for all $\mathfrak{p} \in \operatorname{Ass}(R)$. By lemma 5.6 we then have that $\operatorname{ann}(M) \not \subset \cup_{\mathfrak{q} \in \operatorname{Ass}(R)} \mathfrak{q}$. Note that $\cup_{\mathfrak{q} \in \operatorname{Ass}(R)} \mathfrak{q}$ is the set of all the zero-divisors on $R$. This means that $\operatorname{ann}(M)$ contains a non-zero-divisor. 
Case 2 Suppose $\operatorname{rank}\left(M_{\mathfrak{p}}\right) \neq 0$ for some $\mathfrak{p} \in \operatorname{Ass}(R)$. Then $\operatorname{rank}\left(M_{\mathfrak{p}}\right) \neq 0$ for all $\mathfrak{p} \in \operatorname{Ass} R$ by $(*)$. Since $M_{\mathfrak{p}}$ is a free $R_{\mathfrak{p}}$-module, $\operatorname{ann}_{R_{\mathfrak{p}}}\left(M_{\mathfrak{p}}\right)=0$, which implies $(\operatorname{ann}(M))_{\mathfrak{p}}=0$. If $\operatorname{ann}(M) \neq 0$, then $\operatorname{Ass}(\operatorname{ann}(M)) \neq \emptyset$. Take $\mathfrak{q} \in \operatorname{Ass}(\operatorname{ann}(M))$. Since $\operatorname{Ass}(\operatorname{ann}(M)) \subset \operatorname{Ass}(R), \mathfrak{q} \in \operatorname{Ass}(R)$. But $(\operatorname{ann}(M))_{\mathfrak{q}} \neq 0$. This is a contradiction.

Now we are able to answer the question posed at the beginning of this section.

Corollary 5.8. Let $I=(x)$ be a principal ideal generated by a zero-divisor of $R$. Then the $p d_{R}(I)=\infty$.

Proof. Let $M=I$. Since $M$ is generated by a zero-divisor then $\operatorname{ann}(M) \neq 0$. Also, $\operatorname{ann}(M)$ does not contain any non-zero-divisors since every element of $\operatorname{ann}(M)$ is annihilated by $x$. Therefore by the contrapositive of theorem 5.7, $M$ must have infinite projective dimension. 


\section{References}

[1] Winifried Bruns, H. Jurgen Herzog, Cohen-Macaulay Rings, Cambridge Studies in Advanced Mathematics.

[2] Luchezar Avramov, Infinite Free Resolutions, http://www.math.unl.edu/ lavramov2/papers/resolution.pdf, Purdue University

[3] Hideyuki Matsumura and Miles Reid, Commutative Ring Theory, Cambridge University Press 1989.

[4] Shiro Goto and Naoyoshi Suzuki, What makes $\operatorname{Tor}_{1}(I, R / I)$ free?, Proc. Amer. Math Soc., 1991, 605-611.

[5] Joseph J. Rotman, An Introduction to Homological Algebra, Springer 2009. 